\title{
UTERINE RUPTURE DURING PREGNANCY - CASE REPORT
}

\author{
Mihai Cristian Dumitrașcu ${ }^{1,2}$, Răzvan Fodoroiu ${ }^{2}$, George Cătălin Nenciu ${ }^{1}$, Aida Petca ${ }^{1,3}$, Răzvan \\ Petca $^{1,4}$, Flori Șandru ${ }^{1,3}$, Monica Mihaela Cîrstoiu ${ }^{1,2}$ \\ ${ }^{1}$ Carol Davila University of Medicine and Pharmacy, Bucharest, Romania \\ ${ }^{2}$ University Emergency Hospital Bucharest, Romania \\ ${ }^{3}$ University Emergency Hospital Elias, Bucharest, Romania \\ ${ }^{4}$ Hospital Professor Dr. Theodor Burghele, Bucharest, Romania
}

\section{CASE REPORT Abstract}

Doi: $10.33695 /$ rojes.v2i2.32 Accepted: 27.09 .2020
Corresponding author:

Flori Șandru

florysandru@yahoo.com
Uterine rupture is a life-threatening complication in pregnancy with no specific signs and symptoms, that requires fast diagnosis and surgical treatment. The main risk factors for uterine rupture are previous caesarean section deliveries and myomectomies, which can lead to improper uterine wall healing. We report a case of a 37-year-old secundigravida in 29 weeks of pregnancy, with prior caesarian delivery that was admitted in our ward for altered general status and abdominal pain. The patient underwent emergency laparotomy during which we found a massive $3500 \mathrm{ml}$ hemoperitoneum, $1000 \mathrm{~g}$ dead fetus and a rupture in the posterolateral uterine wall. The uterine scar from the previous caesarian section was intact. Uterine rupture is a complication with a growing incidence in past years that is very difficult to predict, which can occur at any time during pregnancy, especially during labour, but also in the second or early third trimester. Fast diagnosis and proper management is imperious because of the catastrophic outcomes uterine rupture is associated with.

Keywords: uterine rupture, pregnancy, caesarean section, risk factors

\section{Introduction}

Uterine rupture during pregnancy represents a severe complication, most of the time leading to catastrophic outcomes. It is highly associated with maternal and fetal morbidity and has become more frequent in recent years because of the increasingly caesarean section deliveries. It represents the spontaneous tearing of the uterine wall that may result in the fetus being pushed out into the peritoneal cavity [1]. It can occur in women with a surgical scar from previous surgery, or in a native, unscarred uterus. In some cases, this event leads to peripartum hysterectomy, shock and hemorrhage [2,3].

\section{Case presentation}

We report a case of a 37-year-old secundigravida in 29 weeks of pregnancy, with prior caesarian delivery that was admitted in our ward for altered general status and abdominal pain. Clinically, the patient was unconscious, blood pressure 40/20 $\mathrm{mmHg}$, with no vaginal bleeding and severe fetal bradycardia. The patient underwent emergency laparotomy during which we found a massive $3500 \mathrm{ml}$ hemoperitoneum, $1000 \mathrm{~g}$ dead fetus and a rupture in the posterolateral 
uterine wall. The uterine scar from the previous caesarian section was intact. Because of the massive uterine bleeding and incoagulable PT-INR, an emergency hysterectomy was performed. Figures 1-3 show intraoperative uterine rupture and suture. Figure 4 shows excised uterus. The patient was discharged from the hospital after 5 days with good general status and no post-surgical complications.

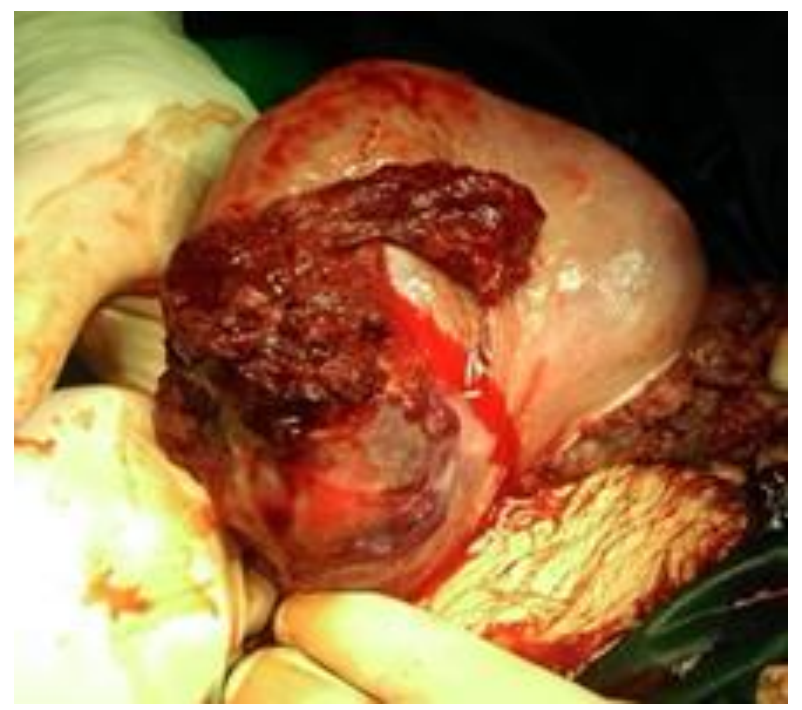

Figure 1 - Intraoperative image of uterine rupture

\section{Discussion}

Uterine rupture on the unscarred pregnant uterus is a very rare event, which is estimated to occur in 1/20,000 pregnancies $[4,5]$. In literature there are reported over 2000 cases of uterine rupture in 3.000 .000 pregnancies with an incidence of $0.07 \%$. In recent years, the use of caesarean section deliveries has increased from $6.7 \%$ in 1990 to $19.1 \%$ in 2014 globally [6]. As a consequence, the number of deliveries by women with a prior caesarean section is also increasing. Studies show that the risk of uterine rupture is 50 times higher among patients with a scarred uterus [7]. The incidence of uterine rupture after transversal incision is considered to be $0,2-1,5 \%$ while on median incisions, the incidence is 4-9\%. Maternal mortality is considered to be between 1-13\% with a high perinatal $74-92 \%$ mortality.

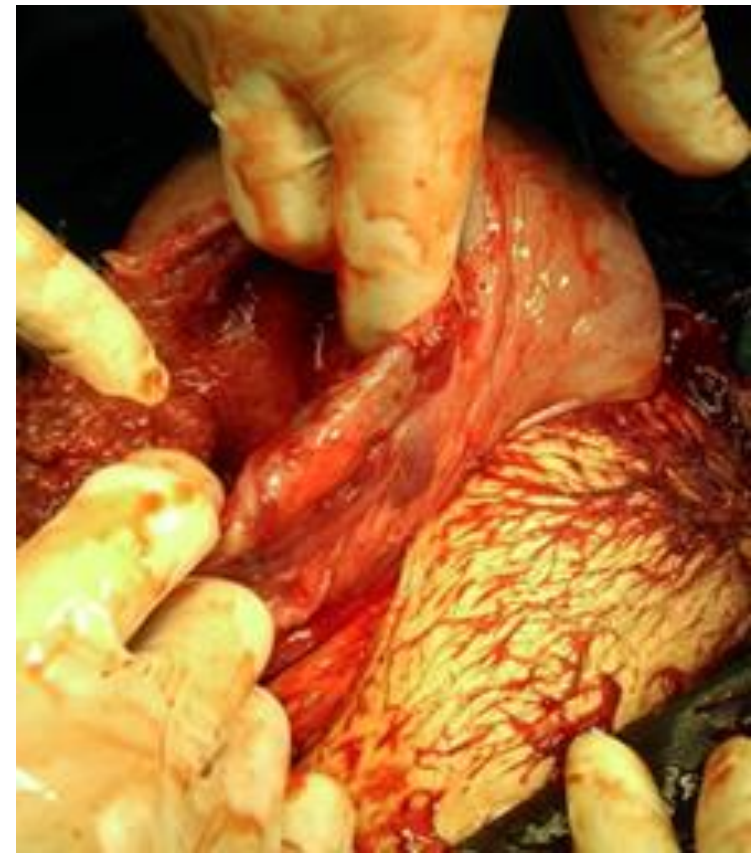

Figure 2 - Intraoperative image of uterine rupture

A systematic review performed by the World Health Organization has established that the prevalence of uterine rupture is lower in developed countries than in developing countries [8]. According to a study on uterine rupture in developing countries, the prevalence of women with prior caesarean section and other uterine surgeries among patients with uterine rupture was over $60 \%$ [9].

Regarding recommendations from The Society of Obstetricians and Gynecologists of Canada (SOGC) guideline, it is considered that although data is limited, routine caesarean section delivery is recommended for patients who previously underwent myomectomy that included a great portion of the uterus. Some recommend caesarean section delivery for patients who underwent myometrectomy, but general recommendations should be individualized and should be given by the physician who performed the first surgery. Uterine rupture after myomectomy is highly 
associated with the absence of suture in multiple layers during intramural myomectomy or with the excessive use of electrocoagulation procedures [10].

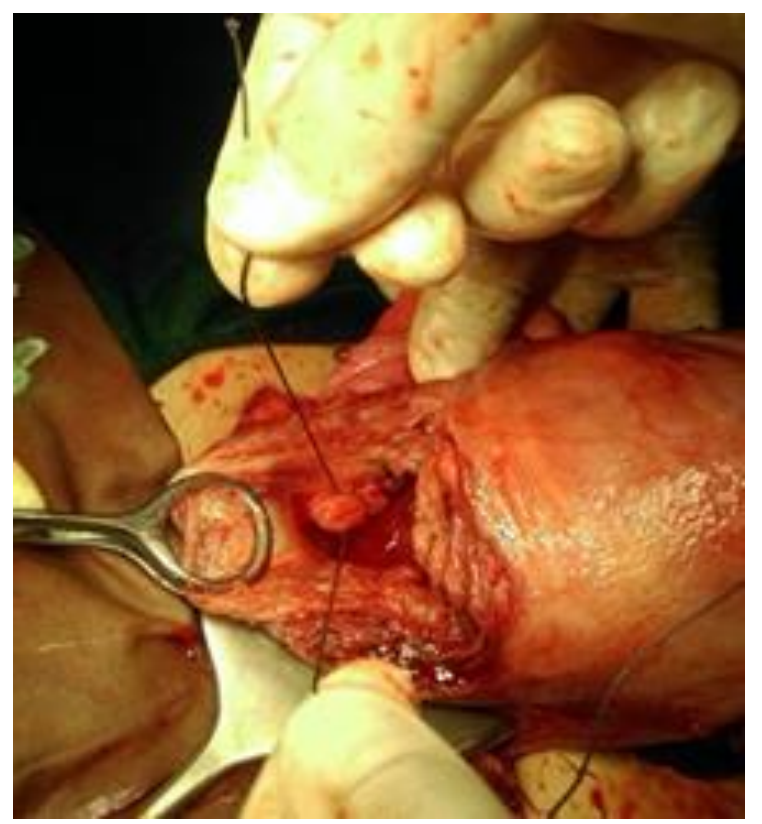

Figure 3 - Intraoperative suturing of the uterus

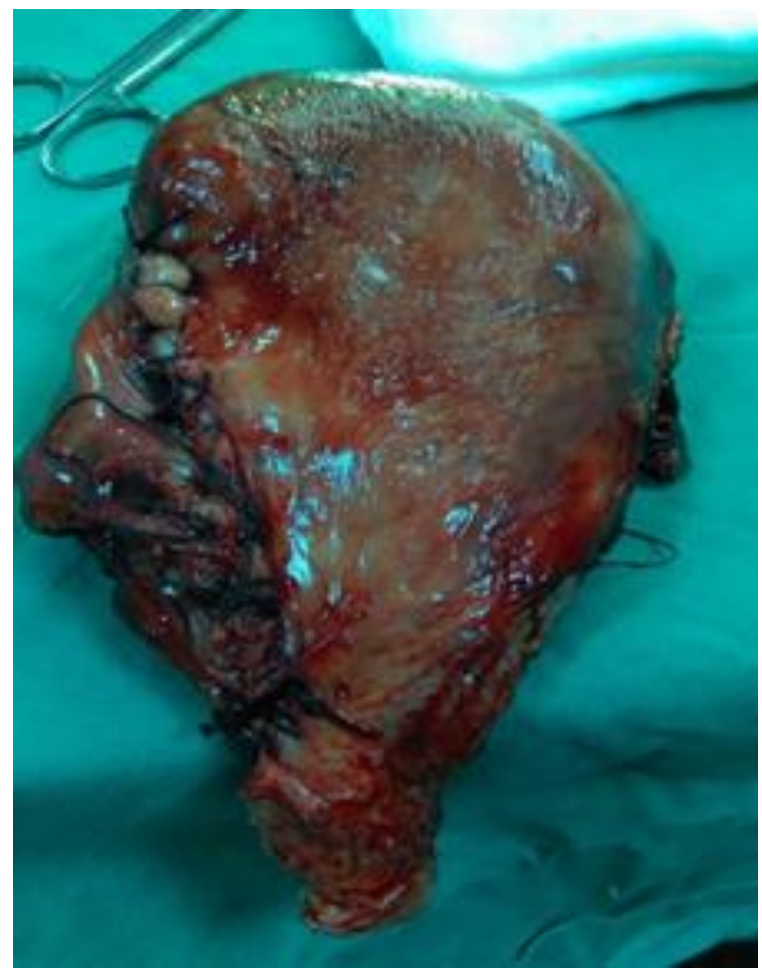

Figure 4 - Excised uterus
The severity of symptoms during uterine rupture in pregnancy is closely related to the timing and the extent of uterine defect. Uterine rupture on an unscarred uterus is more violent and can have a greater hemodynamic impact than a rupture occurring on a previous uterine scar. This can be explained because of the reduced vascularity in the scarred tissue. Because of the catastrophic effect of the uterine rupture, a fast diagnosis must be done. The most common signs and symptoms are fetal heart rate anomalies, maternal and fetal bradycardia, blood pressure drop, uterine hyperstimulation, loss of uterine tonus or ceasing of contractions, abnormal labor, abdominal pain, vaginal bleeding and shock. When these signs and symptoms are present, the doctor should perform transabdominal ultrasonography. Rozenberg et al studied the thickness of the lower uterine segment in pregnant women at 36-38 weeks of gestational age. The results showed that the risk of uterine rupture is significantly greater if the uterine wall is thinner than $3.5 \mathrm{~mm}$. Using this value as a cutoff, the authors had an $88 \%$ sensitivity and a $73 \%$ specificity in predicting subsequent uterine rupture [11].

Myomectomy is also one major risk factor for uterine rupture. Similarities regarding the prevalence of uterine rupture among patients with caesarean section and myomectomy have been described. One literature review that included 1000 patients after prior myomectomy revealed an overall uterine rupture incidence of $0.93 \%$ [12]. Fibroid location before a myomectomy can influence the risk of uterine rupture. Intramural fibroids have a greater risk of developing a rupture probably because of the full thickness of the uterus requiring surgical repair. One study reporting 2367 pregnancies after prior myomectomy reviewed that there is no difference in the incidence of uterine rupture between abdominal myomectomy and laparoscopic approach [12]. In order to repair the uterine incision, meticulous closure is needed. This can be more difficult via a 
laparoscopic approach and can influence the healing process. One study reported a higher incidence of rupture in laparoscopic myomectomy [13] which can be explained by the fact that hemostasis is performed using bipolar coagulation and not classic sutures as in abdominal myomectomy. The thermal damage of the myometrium induces connective tissues modifications that cannot later remodel during pregnancy and can further lead to rupture. Other risk factors to be taken into consideration are the number and size of the fibroids removed, potential infection, hematoma that develops within the myometrium, hemostasis and suturing technique of the myometrial defect and the individual characteristics of the patients' healing process [13]. One interesting aspect concluded in one study is that almost $70 \%$ of uterine ruptures occurred in women who received a double-layer suture versus 30\% who received a single-layer suture during myomectomy [12]. In some cases, symptoms of uterine rupture can be masked by postsurgical intestinal adhesions covering and occluding the rupture. An article by Blihovde et al. showed that uterine rupture should be considered in patients with prior uterine surgeries that associate abdominal pain, even without evidence of hypovolemia, vaginal bleeding, contractions or fetal heart rate anomalies [14].

Main risk factors for uterine rupture include grand multipara, previous uterine scars, obstructed labor, obstetric trauma, misuse of uterine stimulants, severe concealed accidental hemorrhage, anterior sacculation or posterior sacculation, external trauma.

Another risk factor for uterine rupture is considered to be the use of induction agents, such as prostaglandins and oxytocin. These agents are used to increase uterine contractions and ripen the cervix. Prostaglandins are naturally occurring hormone-like substances that stimulate changes in the cervix that eventually cause it to ripen. In the end, these agents lead to hyperstimulation of the uterus, which can weaken previous scars and subsequently increase the risk of scar opening during labor [15]. One study confirmed that the induction of labor doubled the risk of uterine rupture in comparison with spontaneous labor. The study also showed that uterine rupture can occur in induced labor even on the unscarred uterus [16].

Zeteroglu et all. presented in 2017 a case series with three patients that had hysteroscopic transcervical resection of the uterine septum or laparoscopic myomectomy and within one year of the procedure, they remained pregnant. They presented uterine rupture at 10, 22 and 38 weeks of gestation [17]. Ergenoglu et all also estimated that the rate of uterine rupture after these procedures was $1-2,7 \%$. Also, the risk for uterine rupture in the first trimester is higher in patients who previously presented uterine rupture during another pregnancy [18].

Other risk factors include maternal age ( $\geq 35$ years) that can double the risk of uterine rupture, uterine malformation, macrosomia, malpresentation, fetal anomalies, uterine perforation, open fetal surgery, obstructed labor, multiparity or grand multiparity, trauma, placenta accreta and percreta [19].

Our case of uterine rupture is a rare case of rupture in the second trimester, when uterine rupture is not to be considered with the highest risk. No certain cause was found and we consider that this life-threatening pregnancy complication is highly unpredictable and immediate surgical treatment must be performed. In most cases, hysterectomy may be the only solution, because suturing of the uterus rupture may later develop serious complications. Although in most of the cases the fetus does not survive, because of the high mortality of this emergency, saving the mother's life is a medical and surgical accomplishment. 


\section{Conclusions}

Uterine rupture is a complication with a growing incidence in past years that is very difficult to predict, which can occur at any time during pregnancy, especially during labor, but also in the second or early third trimester. Myomectomy is a major risk factor for uterine rupture, in some cases not only causing the rupture itself but also masking it through postsurgical intestinal adhesions and thus preventing its early diagnosis. Despite the advantages of the laparoscopic approach for myomectomy, it is associated with a higher risk of uterine rupture because of the use of thermal hemostasis. Hysteroscopic surgeries are also described in the literature but with few cases reported. Fast diagnosis and proper management is imperious because of the catastrophic outcomes uterine rupture is associated with.

\section{Bibliography}

[1] Chuni. Analysis of uterine rupture in a tertiary center in Eastern Nepal: lessons for obstetric care. J Obstet Gynaecol Res. 2006;32(6):574-579.

[2] I Ozdemir, N Yucel, O Yucel. Rupture of the pregnant uterus: a 9-year review. Arch Gynecol Obstet. 2005;272(3):229-231.

[3] M Al Sakka, A Hamsho, L Khan. Rupture of the pregnant uterus--a 21-year review. Int J Gynaecol Obstet. 1998;63(2):105-108.

[4] M Dow, JR Wax, MG Pinette, et al. Thirdtrimester uterine rupture without previous cesarean: a case series and review of the literature. Am J Perinatol 2009; 26:739.

[5] DA Miller, TM Goodwin, RB Gherman, RH Paul. Intrapartum rupture of the unscarred uterus. Obstet Gynecol 1997; 89:671.

[6] J. PVogel,. et al. Use of the Robson classification to assess caesarean section trends in 21 countries: a secondary analysis of two WHO multicountry surveys. Lancet Glob Health. 3, e260-270,
[7] M Kaczmarczyk, P Sparén, P Terry, S Cnattingius. Risk factors for uterine rupture and neonatal consequences of uterine rupture: a population-based study of successive pregnancies in Sweden. BJOG 2007; 114: 1208- 14.

[8] G. J.Hofmeyr, L Say. \& A. M Gulmezoglu. WHO systematic review of maternal mortality and morbidity: the prevalence of uterine rupture. BJOG. 112, 1221-1228,

[9] Y Berhe. \& L. L Wall. Uterine rupture in resource-poor countries. Obstet. Gynecol. Surv. 69, 695-707

[10] Society of Obstetricians and Gynaecologists of Canada (SOGC) Guidelines for vaginal birth after previous cesarean birth: N 155 (2005)J ObstetGynaecol Can pp164174

[11] PRozenberg, F Goffinet, HJ Philippe, I Nisand. Thickness of the lower uterine segment: its influence in the management of patients with previous cesarean sections. Eur J Obstet Gynecol Reprod Biol. 1999 Nov. 87(1):39-45.

[12] Z Gambacorti-Passerini, AC Gimovsky, A Locatelli, V Berghella. Trial of labor after myomectomy and uterine rupture: a systematic review. Acta Obstet Gynecol Scand 2016;95:724-734.

[13] A Vimercati, V Del Vecchio, A Chincoli, A Malvasi, and E Cicinelli, "Uterine Rupture after Laparoscopic Myomectomy in Two Cases: Real Complication or Malpractice?," Case Reports in Obstetrics and Gynecology, vol. 2017, Article ID 1404815, 5 pages, 2017. [14] L Blihovde, J Tawfik, DA Hill. Prelabor third-trimester uterine rupture in an unscarred uterus with occlusion by fetal small parts: a case report. J Reprod Med. 2010;55(910):437-440.

[15] CS Buhimschi, IA Buhimschi, S Patel, AM Malinow, CP Weiner. Rupture of the uterine scar during term labour: contractility or biochemestry? BJOG 2005:112:38-42. 
[16] JG Smith, DC Merrill. Oxytocin for induction of labor. Clin Obstet Gynecol 2006:49:594-608.

[17] Ş Zeteroğlu M, Aslan, B Akar, R Ada Bender, A Başbuğ, E Çalışkan. Uterine rupture in pregnancy subsequent to hysteroscopic surgery: A case series. Turk J Obstet Gynecol. 2017;14(4):252-255.
[18] M Ergenoglu, AO Yeniel, N Yıldırım, A Akdemir, S Yucebilgin. Recurrent uterine rupture after hysterescopic resection of the uterine septum. Int J Surg Case Rep. 2013;4(2):182-184.

[19] E Lieberman. Risk factors for uterine rupture during a trial of labor after cesarean. Clin Obstet Gynecol. 2001 Sep. 44(3):609-21. 